\title{
EDITORIAL Vol. 19, Núm. 2
}

En esta edición de la revista PALOBRA, Palabra que Obra, aspiramos a continuar con el proceso de indexación a sistemas de información internacional, y seguir manteniendo la calidad editorial que dé cuenta de nuestro compromiso en la popularización de conocimiento científico de autores nacionales e internacionales.

Para esta decimonovena edición, se recepcionaron 82 artículos, dentro de los cuales luego de su evaluación externa bajo la modalidad doble ciego por pares académicos reconocidos, se seleccionaron con rigurosidad 40, distribuidos así: 21 artículos para el Número uno ( $\left.\mathrm{N}^{\circ} 1\right)$ y 19 para el Número 2 ( $\left.{ }^{\circ} 2\right)$ del volumen 19. El Volumen 19 - Número 2 cuenta con 7 de investigación científica y tecnológica y 12 de reflexión, relacionados con categorías de análisis de diversas temáticas del área de las ciencias sociales interdisciplinar: 1. Construcción de paz y derechos humanos, 2. Género, 3. Trabajo Social, 4 Comunicación Social, 5. Migraciones, 6. Educación, Currículo y Practicas pedagógicas y 7 . Construcción y practicas sociocultural.

El primer eje temático, Construcción de paz y derechos humanos, lo integran dos artículos, que resaltan por un lado una de las razones por las cuales se ha dificultado los procesos de construcción de paz en Colombia, y por el otro, la participación como una herramienta para garantizar el goce efectivo de los derechos humanos.

Inicialmente se encuentra el artículo "RECONOCIMIENTO Y PROCESO DE PAZ EN COLOMBIA" enviado por Luis Zúñiga Herazo y Harold Valencia López en el que analizan los alcances de la Teoría de la Lucha por el Reconocimiento de Axel Honneth, en el marco de los acuerdos de paz entre el gobierno colombiano y la guerrilla de las FARC. Para ello muestran cómo el reconocimiento constituye un presupuesto previo para el proceso de paz desarrollado en Colombia; asimismo plantean que la degradación y la prolongación del conflicto social y armado ha dificultado su superación.

Por su parte las autoras, Hingrid Camila Pérez Bermúdez y Angie Marcela Santana De Ángel proponen el artículo "RESISTENCIAS Y REIVINDICACIONES SOCIALES. CONTEXTUALIZACIÓN DEL AMPARO A LOS DERECHOS HUMANOS DESDE $L A S$ COMUNIDADES", que manifiesta la intención de deconstruir la ley y forjarla desde abajo, desde las comunidades, desde sus identidades, desde lo que hace parte del ser; de esta forma, se proclama la salvaguarda de los derechos humanos y su efectividad desde la teoría crítica del derecho alternativo. 
El segundo eje temático Género, incluye 2 artículos que problematizan las conflictividades presentes en el inicio de la sexualidad en la mujer y en la asunción de roles cultural e históricamente definidos respecto a la maternidad que, en algunos casos condicionan el acceso a oportunidades de desarrollo personal.

El primer artículo "ADOLESCENCIA, SEXUALIDAD Y REPRODUCCIÓN: TRES DIMENSIONES FUNDAMENTALES PARA LA COMPRENSIÓN DEL FENÓMENO DEL EMBARAZO ADOLESCENTE" realizado por Ana Cecilia Ojeda Avellaneda y Leticia Montero Torres se analizan los significados alrededor del embarazo como una situación social de alto impacto individual, familiar y cultural, destacando el peso del embarazo como una representación conflictiva especialmente para las adolescentes en condiciones económicas desfavorables.

En el segundo artículo de este eje, la autora Laury Katherine Julio Muñoz en el artículo titulado "MATERNIDAD: OPCIÓN DE VIDA O IMPOSICIÓN SOCIAL" se plantea reestructurar el discurso en relación a la mujer el cual está directamente relacionado con la maternidad, puesto que el concepto de la familia ha cambiado con el tiempo, y el rol de la mujer en la sociedad se ha diversificado, ampliando un abanico de opciones que han permitido ver a la mujer en muchas otras facetas.

En el tercer eje temático Trabajo Social, se encuentra el artículo "SISTEMATIZACIÓN DEL PROCESO DE FORMACIÓN DE TRABAJADORAS SOCIALES EN EL MARCO DE LAS PRÁCTICAS INSTITUCIONALES: UNA APUESTA A LA CONSOLIDACIÓN DEL PROYECTO ÉTICO POLÍTICO PROFESIONAL" escrito por Alba Lucía Marín Rengifo Jhon Sebastián Aguirre Cano y Sandra Yaneth Vallejo González, que muestra una reconstrucción participativa de la experiencia vivida por las orientadoras de práctica, asesores institucionales, estudiantes y egresadas del programa de Trabajo Social de la Universidad de Caldas, como insumo para la consolidación de un proyecto ético político profesional en la Universidad, desde las concepciones de práctica institucional develadas.

En el cuarto el eje temático Comunicación Social, se integraron 2 artículos que reivindican la comunicación como estrategia para facilitar procesos de participación social en las comunidades locales y procurar el desarrollo social y territorial.

Las autoras Mercedes Posada Meola y Mary Nieto Ariza, en el articulo "PRÁCTICAS Y SABERES DE LA COMUNICACIÓN PARA EL CAMBIO SOCIAL EN LA EXPERIENCIA DE FUNSAREP" plantean como una comunicación dialógica, participativa, horizontal y orientada al cambio, es capaz de incidir de manera sostenida en los procesos de formación, autogestión, empoderamiento y transformación que lideran las comunidades organizadas para incidir en su desarrollo.

Y el segundo artículo denominado "IMPLICACIONES Y RETOS PARA LA FORMACIÓN EN 
COMUNICACIÓN PARA EL CAMBIO SOCIAL. NUEVAS LÍNEAS DE ABORDAJE escrito por Daivy Dalila Díaz Santana, Giovanni Bohórquez Pereira y Olga Beatriz Barrios Rueda, se centra en los campos de la cultura digital con sus nuevas narrativas, la innovación social, la gobernanza y el deporte, desde los cuales se vienen abordando alternativas para generar cambios en la sociedad y se trazan caminos para generar competencias para el análisis, la planeación y la acción de la comunicación en relación con los procesos de desarrollo social y territorial.

En el quinto eje temático Migraciones, los autores Carlos Yánez Canal y Andrés Yáñez Chavarriaga en su artículo titulado "LA TRANSNACIONALIDAD DEL ESTUDIANTE MIGRANTE COLOMBIANO: HACIA LA RECONFIGURACIÓN DE SUS IDENTIDADES” encontraron que, el sentido y significado que le atribuyen profesionales colombianos que regresaron a Colombia después de realizar estudios en el exterior, a sus experiencias y vivencias permite dilucidar los cambios suscitados en ellos desde el punto de vista transnacional, lo que implica la reconfiguración de sus identidades.

El sexto eje temático Educación, Currículo y Practicas pedagógicas, lo conforman 10 artículos de reflexión e investigación que versan sobre los elementos que fundamentan la apuesta metodológica y didáctica del modelo pedagógico en el contexto actual, así como en la problematización de categorías como educación, currículo, prácticas pedagógicas, la investigación formativa, la innovación educativa, tanto en la educación básica y secundaria como en la formación de educadores. Asimismo, abren el debate sobre la practica pedagógica como un escenario en el cual se construyen aprendizajes, utilizando herramientas como las lúdicas virtuales, las expresiones metafóricas y lingüísticas, los lenguajes de la música, la danza, los ritmos, la narrativa de los versos entre otros.

El primer artículo ¿QUÉ SIGNIFICA EDUCAR A LA PRIMERA INFANCIA EN LA POSTMO$D E R N I D A D$ ?, las autoras Mery Luz Pacheco Bohórquez y Mónica Patricia Borjas afirman que la concepción de infancia, así como la comprensión del por qué y para qué educar, se constituyen en preguntas básicas y trascendentales que fundamentan la apuesta metodológica y didáctica, la cual se inscribe en un modelo pedagógico determinado.

En el segundo el autor Rodrigo Arenas Carter en su artículo titulado "PERSPECTIVAS SOBRE LA INCLUSIÓN DE LA PERFORMANCE EN LA EDUCACIÓN ARTÍSTICA ESCOLAR SECUNDARIA EN LATINOAMÉRICA", presenta una reflexión en torno a la inserción de la performance como contenido en el currículum básico de la Educación Artística Escolar a nivel secundario en Latinoamérica.

El tercer artículo "PEDAGOGÍA INFANTIL Y PRÁCTICAS PEDAGÓGICAS: CASO CONVENIO UNIVERSIDAD DE CARTAGENA- UNIVERSIDAD DEL TOLIMA, DURANTE 2013-2016” Nelfy Castro Estremor, Diana Castro Campo y Liliana Durango Llorente presenta algunas 
reflexiones sobre los impactos positivos de los procesos de prácticas que se han llevado a cabo en el programa de Licenciatura en Pedagogía Infantil, ofertado por la Facultad de Ciencias Sociales y Educación de la Universidad de Cartagena, en convenio con la Universidad del Tolima (20132016).

En el cuarto, los autores José Eriberto Cifuentes Medina, Jaime Andres Torres Ortiz y Leidy Carolina Plazas Díaz en el artículo titulado "REFLEXIONES DEL DESARROLLO PEDAGÓGICO SOCIO-HUMANÍSTICO DE ESTUDIANTES EN PROCESO DE FORMACIÓN DOCENTE DE BÁSICA PRIMARIA" presentan una reflexión sobre la importancia de los conocimientos socio-humanísticos en procura del rescate de la esencia de la academia, así como en la formación integral e idónea de estudiantes en proceso de formación docente de Educación Básica Primaria, con el fin de generar procesos democráticos y ciudadanos, así como espacios interculturales que permitan transformaciones de pensamiento necesarias para humanizar las sociedades de hoy.

El quinto artículo "TRANSFERENCIA DE CONOCIMIENTO E INVESTIGACIÓN FORMATIVA: LECCIONES APRENDIDAS Y DESAFÍOS PARA LOS SEMILLEROS DE INVESTIGACIÓN" escrito por Oscar Mauricio Gómez Miranda, María Eugenia Morales Rubiano y Paola Andrea Plata Pacheco, en donde se identificaron las lecciones aprendidas y desafíos en los procesos de transferencia de conocimiento y formación de capacidades a partir de la experiencia de un semillero de investigación de la Universidad Militar Nueva Granada.

En el sexto, Paula Andrea Mora Pedreros, Manuel Santiago Pajajoy y Deyser Gutiérrez en el artículo denominado "EL OBSERVATORIO FILOSÓFICO VIRTUAL COMO UNA COMUNIDAD DE APRENDIZAJE DESDE LA LUDIFICACIÓN" evidenciando que la filosofía requiere de herramientas para la innovación educativa tales como el uso de lúdicas digitales, que logren un impacto significativo en la didáctica.

El séptimo artículo titulado "UNA CARACTERIZACIÓN DEL LENGUAJE METAFÓRICO DOCENTE Y SU DIVERGENCIA CON EL ENTENDIMIENTO DE LOS ESTUDIANTES RESPECTO DEL CONCEPTO DE NÚMERO RACIONAL presentado por Oscar Fernández Sánchez y Cristian David Franco Restrepo, se indaga sobre el discurso de los docentes y lenguaje metafórico utilizado en el aula para la enseñanza de los números racionales, así como las posibles divergencias entre este lenguaje y el entendimiento de los estudiantes.

Por su parte, en el octavo artículo, Arlex Berrio Peña en su artículo "RESIGNIFICACIÓN PEDAGÓGICA: REINVENCIÓN DE LA EDUCACIÓN" analiza la importancia y sentido de la resignificación pedagógica, desde el estudio de su relación con las prácticas docentes, el currículo, la didáctica y el papel de la familia en el proceso educativo, así como de la importancia de la sistematización para un proceso de resignificación pedagógica efectiva. 
En el noveno, Laura Rangel Bernal en su artículo denominado "EDUCACIÓN SEXUAL EN LAS ESCUELAS MEXICANAS: ENTRE EL DISCURSO CIENTÍFICO LAICO Y LA MORAL RELIGIOSA" presenta que, la enseñanza de los contenidos de sexualidad tiene indicios de una moral sexual vinculada al catolicismo y que dicho sesgo no sólo afecta el cumplimiento de los objetivos de la educación sexual, sino que, además contravienen el principio de laicidad que rige a la educación en México.

Y el décimo artículo de este eje, corresponde al artículo titulado "EL SON DE NEGRO UN DISENO DE INTEGRACIÓN CURRICULAR PARA SANTA LUCÍA ATLÁNTICO Y LA SUBREGIÓN CANAL DEL DIQUE EN EL CARIBE COLOMBIANO” escrito por Manuel Antonio Pérez Herrera Muestra un diseño de integración curricular resultado de un proceso de búsqueda de nuevas prácticas y saberes inculturada en la cultura ancestral Son de Negro, contexto visibilizado como problema educativo-formativo en la localidad de Santa Lucia y su área de influencia, como una oportunidad para oportunidad de fomentar la conciencia del sentido de identidad y trabajar en forma integrada para resolver desde la escuela problemas del contexto.

Finalmente, en el eje temático Construcción y practicas sociocultural, se encuentra el artículo denominado "SINCRETISMO RELIGIOSO EN LAS FIESTAS DE LA VIRGEN DE GUADALUPE DE CATAMBUCO, VESTIGIOS DE LA COLONIZACIÓN ESPAÑOLA” escrito por Alba Yamile Timaná de la Cruz, Hugo Horacio Rojas Achicanoy y Carlos Alfredo Muñoz, se identifican las manifestaciones de devoción a la imagen de la Virgen de Guadalupe, a través de diversas expresiones culturales y artísticas realizadas en su homenaje como una muestra de amor y agradecimiento por los favores recibidos, lo cual evidencia el sincretismo religioso, como una forma de resistencia al desarraigo cultural de los antepasados, en Pasto, Colombia.

Por último, expresarles que el equipo editorial se encuentra trabajando para incrementar el impacto y el posicionamiento nacional e internacional de la revista; para ello, ha participado activamente en el curso "Currículo del Editor" impartido por el Ministerio de Ciencia, Tecnología e Innovación y ha diseñado un plan de acción a corto, mediano y largo plazo a fin de cumplir con los parámetros y estándares de gestión editorial y mantener tanto la calidad de nuestra Revista digital así como su visibilidad. 